\title{
Predictions from Quantum Cosmology
}

\author{
Alexander Vilenkin*) \\ Institut des Hautes Etudes Scientifiques \\ 35 , route de Chartres \\ 91440 Bures-sur-Yvette \\ FRANCE
}

\begin{abstract}
The world view suggested by quantum cosmology is that inflating universes with all possible values of the fundamental constants are spontaneously created out of nothing. I explore the consequences of the assumption that we are a "typical" civilization living in this metauniverse. The conclusions include inflation with an extremely flat potential and low thermalization temperature, structure formation by topological defects, and an appreciable cosmological constant.
\end{abstract}

*) On leave from Tufts University

e-mail address: AVILENKI@PEARL.TUFTS.EDU 
Why do the constants of Nature take the particular values that they are observed to have in our universe? It certainly appears that the constants have not been selected at random. Assuming that the particle masses are bounded by the Planck mass $m_{p}$ and the coupling constants are $\underset{\sim}{1}$, one expects that a random selection would give all masses $\sim m_{p}$ and all couplings $\sim 1$. The cosmological constant would then be $\Lambda \sim m_{p}^{2}$ and the corresponding vacuum energy $\rho_{v} \sim m_{p}^{4}$. In contrast, some of the particle masses are more than 20 orders of magnitude below $m_{p}$, and the actual value of $\rho_{v}$ is $\underset{\sim}{<} 10^{-120} m_{p}^{4}$. (I use the system of units in which $\hbar=c=1$.)

It has been argued [1] that the values of the constants are, to a large degree, determined by anthropic considerations: these values should be consistent with the existence of conscious observers who can wonder about them. If one assumes that the production of heavy elements in stars and their dispersement in supernova explosions are essential for the evolution of life, then one finds that this Anthropic Principle imposes surprisingly stringent constraints on the electron, proton and neutron masses $\left(m_{e}, m_{p r}\right.$ and $\left.m_{n}\right)$, the $W$-boson mass $m_{W}$, and the fine structure constant $e^{2}$. An anthropic bound on the cosmological constant can be obtained by requiring that gravitationally bound systems are formed before the universe is dominated by the vacuum energy [2].

I should also mention the popular view that there exists a unique logically consistent Theory of Everything and that all constants can in principle be determined from that theory. The problem, however, is that the constants we observe depend not only on the fundamental Lagrangian, but also on the vacuum state, which is likely not to be unique. For example, in higher-dimensional theories, like superstring theory, the constants in the 
four-dimensional world depend on the way in which the extra dimensions are compactified. Moreover, Coleman has argued [3] that all constants appearing in sub-Planckian physics become totally undetermined due to Planck-scale wormholes connecting distant regions of spacetime.

Finally, it has been suggested that the explanation for the values of some constants can be found in quantum cosmology. The wave function of the universe gives a probability distribution for the constants which can be peaked at some particular values [4]. Wormhole effects can also contribute an important factor to the probability [5]. Smolin [6] has argued that new expanding regions of the universe may be created as a result of gravitational collapse due to quantum gravity effects. Assuming that the constants in these "daughter" regions deviate slightly from their values in the "mother" region, he conjectured that the observed values of the constants are determined by "natural selection" for the values that maximize the production of black holes. Some problems with this conjecture have been pointed out in Ref. [7].

In this paper I would like to suggest a different approach to determining the constants of Nature. This approach is not entirely new and has elements of both anthropic principle and quantum cosmology. However, to my knowledge, it has not been clearly formulated and its implications have not been systematically explored. My approach is based on the picture of the universe suggested by quantum cosmology and by the inflationary scenario. In this picture, small closed universes spontaneously nucleate out of nothing, where "nothing" refers to the absence of not only matter, but also of space and time [8]. All universes in this metauniverse are disconnected from one another and generally have different values for 
some of the constants. This variation may be due to different compactification schemes, wormhole effects, etc. We shall not adopt any particular hypothesis and keep an open mind as to which constants can be varied and what is the allowed range of their variation.

After nucleation, the universes enter a state of inflationary expansion. It is driven by the potential energy of a scalar field $\varphi$, while the field slowly "rolls down" its potential $V(\varphi)$. When $\varphi$ reaches the steep portion of the potential at some $\varphi \sim \varphi_{*}$, its energy thermalizes, and inflation is followed by the usual radiation-dominated expansion. The evolution of $\varphi$ is influenced by quantum fluctuations, and as a result thermalization does not occur simultaneously in different parts of the universe. In many models it can be shown that at any time there are parts of the universe that are still inflating $[9,10]$. Such eternally inflating universes have a beginning, but have no end.

We are one of the infinite number of civilizations living in thermalized regions of the metauniverse. Although it may be tempting to believe that our civilization is very special, the history of cosmology demonstrates that the assumption of being average is often a fruitful hypothesis. I call this assumption the Principle of Mediocrity. We shall see that, compared to the traditional point of view, this principle gives a rather different perspective on what is natural and what is not.

The Principle of Mediocrity suggests that we think of ourselves as a civilization randomly picked in the metauniverse. Denoting by $\alpha_{i}$ the constants of Nature that can vary from one universe to another, we can write the corresponding probability distribution as

$$
d \mathcal{P}(\alpha)=Z^{-1} w_{\text {nucl }}(\alpha) \mathcal{N}(\alpha) \prod_{i} d \alpha_{i}
$$


Here, $w_{\text {nucl }}(\alpha) \Pi d \alpha_{i}$ is the probability of nucleation for an inflating universe with a given set of $\alpha_{i}$ in the intervals $d \alpha_{i}, \mathcal{N}(\alpha)$ is the average number of civilizations in such a universe (in its entire history) [11], and $Z$ is a normalization factor. We shall interpret (1) as an $a$ priori probability distribution for $\alpha_{i}$.

The inflating part of the universe can be divided into a quantum region, $V(\varphi)>V_{q}$, where the dynamics of the inflaton field $\varphi$ is dominated by quantum fluctuations, and slowroll region, $V_{*}<V(\varphi)<V_{q}$, where the evolution is essentially deterministic. $\left(V_{*}=V\left(\varphi_{*}\right)\right.$ corresponds to the end of inflation). The values of $V_{*}$ and $V_{q}$ are model-dependent. The inflationary expansion rate is given by $H^{2}=8 \pi V(\varphi) / 3 m_{p}^{2}$ and can be arbitrarily high if $V(\varphi)$ is unbounded from above. In order to extend the validity of the theory up to $V(\varphi) \sim$ $m_{p}^{4}$, one can include one-loop matter corrtections to Einstein's action [12]. This may be adequate if the number $N$ of matter fields is large, $N>>1$. Then it can be shown [13] that the resulting equations have no inflationary solutions for $V(\varphi)>V_{\max } \sim m_{p}^{4} / N$. The inflationary expansion rate is therefore bounded from above [14], $H<H_{\max } \sim m_{p} / \sqrt{N}$. Smaller values of $H_{\max }$ can be obtained in dilatonic and higher-dimensional gravity models, or simply in models where $V(\varphi)$ is bounded from above (e.g., when $\varphi$ is a cyclic variable and has a finite range). Here, we shall assume that, for one reason or another, $H$ is bounded by some $H_{\max }$. Eternal inflation is possible if $V_{\max }>V_{q}$.

Let us first assume that $V_{\max }<V_{q}$ in the whole range of variation of $\alpha_{i}$, so that inflation is finite. Very roughly, we can write

$$
\mathcal{N}(\alpha) \sim \mathcal{V}_{*}(\alpha) \nu_{\text {civ }}(\alpha)
$$


where $\mathcal{V}_{*}$ is the volume of the universe at the end of inflation (that is, the 3 -volume of the hypersurface $V(\varphi)=V_{*}$ ), and $\nu_{\text {civ }}$ is the average number of civilizations originating per unit volume $\mathcal{V}_{*}$. The maximum of $\mathcal{V}_{*}$ is achieved by maximizing the highest value of the potential $V_{\max }$ at which inflation starts and minimizing the slope of $V(\varphi)$ between $V_{\max }$ and $V_{*}$ : the field $\varphi$ takes longer to roll down for a flatter potential.

The cosmological literature abounds with remarks on the "unnaturally" flat potentials required by inflationary scenarios. The slope of the potential is severely constrained by the observed isotropy of the cosmic microwave background. With the Principle of Mediocrity, the situation is reversed: flat is natural! Instead of asking why $V(\varphi)$ is so flat, one should now ask why it is not flatter.

Let us now consider the role of other factors in (1). The calculation of $w_{\text {nucl }}(\alpha)$ is a matter of some controversy. The result depends on one's choice of boundary conditions for the wave function of the universe (see, e.g., $[8,15])$. Here we shall adopt the tunneling boundary condition. Then the semiclassical nucleation probability is proportional to $\exp (-|S|)$, where $S$ is the Euclidean action of the corresponding instanton. In Einstein's gravity, $|S|=\pi m_{p}^{2} / H_{\max }^{2}$, and thus $w_{\text {nucl }}(\alpha)$ favors large values of $V_{\max }$ and is not sensitive to other parameters of the model [16].

An important role in constraining the values of $\alpha_{i}$ is played by the "human factor", $\nu_{\text {civ }}(\alpha)$. We do not know what other forms of intelligent life are possible, but the Principle of Mediocrity favors the hypothesis that our form is the most common in the metauniverse. The conditions required for life of our type to exist (the low-energy physics based on the symmetry group $S U(3) \times S U(2) \times U(1)$, the existence of stars and planets, supernova 
explosions) may then fix, by order of magnitude, the values of $e^{2}, m_{e}, m_{\mathrm{pr}}$ and $m_{W}$, as discussed in Ref. [1]. Anthropic considerations also impose a bound on the allowed flatness of the inflaton potential $V(\varphi)$. If the potential is too flat, then the thermalization temperature after inflation is too low for baryogenesis. The lowest temperature at which baryogenesis can still occur is set by the electroweak scale, $T_{\min } \sim m_{W}$. Hence, if other constraints do not interfere, we expect the universe to thermalize at $T \sim m_{W}$. Specific constraints on $V(\varphi)$ depend on the couplings of $\varphi$ to other fields and can be easily obtained in specific models.

Super-flat potentials required by the Principle of Mediocrity give rise to density fluctuations which are many orders of magnitude below the strength needed for structure formation. This means that the observed structures must have been seeded by some other mechanism. The only alternative mechanism suggested so far is based on topological defects: strings, global monopoles and textures, which could be formed at a symmetry breaking phase transition [17]. The required symmetry breaking scale for the defects is $\eta \sim 10^{16} \mathrm{GeV}$. With "natural" (in the traditional sense) values of the couplings, the transition temperature is $T_{c} \sim \eta$, which is much higher than the thermalization temperature $\left(T_{\mathrm{th}} \sim m_{W}\right)$, and no defects are formed after inflation. It is possible for the phase transition to occur during inflation, but the resulting defects are inflated away, unless the transition is sufficiently close to the end of inflation. To arrange this requires some fine-tuning of the constants. However, the alternative is to have thermalization at a much higher temperature and to cut down on the amount of inflation. Since the dependence of the volume factor $\mathcal{V}_{*}$ on the duration of inflation is exponential, we expect that the gain in the volume 
will more than compensate for the decrease in " $\alpha$-space" due to the fine-tuning. We note also that in some supersymmetric models the critical temperature of superheavy string formation can "naturally" be as low as $m_{W}[18]$.

The symmetry breaking scale $\eta \sim 10^{16} \mathrm{GeV}$ for the defects is suggested by observations, but we have not explained why this particular scale has been selected. The value of $\eta$ determines the amplitude of density fluctuations, which in turn determines the time when galaxies form, the galactic density, and the rate of star formation in the galaxies. Since these parameters certainly affect the chances for civilizations to develop, it is quite possible that $\eta$ is significantly constrained by the anthropic factor $\nu_{\text {civ }}(\alpha)$.

If $\nu_{\text {civ }}$ is indeed sharply peaked at some value of $\eta$ and thus fixes the amplitude of density fluctuations and the epoch of active galaxy formation, then an upper bound on the cosmological constant can be obtained by requiring that it does not disrupt galaxy formation until the end of that epoch. The growth of density fluctuations in a flat universe with $\Lambda>0$ effectively stops at a redshift [19] $1+z \sim\left(1-\Omega_{\Lambda}\right)^{-1 / 3}$, where $\Omega_{\Lambda}=\rho_{v} / \rho_{c}$ and $\rho_{c}$ is the critical density. Requiring that this happens at $z<1$ gives $\Omega_{\Lambda}<0.9$. The actual value of $\Lambda$ is likely to be comparable to this upper bound. Negative values of $\Lambda$ are bounded by requiring that our part of the universe does not recollapse while stars are still shining and new civilizations are being formed. This gives a bound comparable to that for positive $\Lambda$ (by absolute value).

Let us now turn to the case of eternal inflation, $V_{\max }>V_{q}$. The evolution of $\varphi$ is then a stochastic process and can be described by a distribution function $\rho(\varphi, t)$ which satisfies a "diffusion equation" with appropriate boundary conditions at $V(\varphi)=V_{*}$ and $V(\varphi)=V_{\max }$ 
[9,20-23]. In an eternally inflating universe, the volume $\mathcal{V}_{*}$ of the hypersurfaces $V(\varphi)=V_{*}$ is infinite and has to be regulated. The simplest way to do this is to cut it off at some time $t=\tau$ and consider the asymptotic behavior as $\tau \rightarrow \infty$. The time variable $t$ can be defined as the proper time on the congruence of geodesics orthogonal to the initial hypersurface at the "moment of nucleation". Since geodesics tend to diverge during inflation, this propertime gauge should be well defined. If $\rho$ is normalized to the total inflating volume, then in the limit $t \rightarrow \infty$ we have [23] $\rho=F(\varphi) \exp \left(d H_{\max } t\right)$, where $d(\alpha)$ can be interpreted [21] as the fractal dimension of the region expanding at the highest rate $H_{\max }(\alpha), 0<d(\alpha)<3$. The asymptotic form of $\mathcal{V}_{*}$ at large $\tau$ is then

$$
\mathcal{V}_{*}(\alpha, \tau)=\tilde{\mathcal{V}}(\alpha) \exp \left[d(\alpha) H_{\max }(\alpha) \tau\right]
$$

and it is clear that in the limit $\tau \rightarrow \infty$ the distribution (1) selects the values of $\alpha_{i}$ that maximize the product $d(\alpha) H_{\max }(\alpha)$,

$$
B(\alpha) \equiv d(\alpha) H_{\max }(\alpha)=\max .
$$

Generically, a function attains its absolute maximum at a single point. If this is so for $B(\alpha)$, then Eq.(4) is sufficient to determine all constants of Nature. However, it is conceivable that the maximum of $B(\alpha)$ is degenerate, so that (4) defines a surface in the space of $\alpha_{i}$. All values not on this surface have a vanishing probability, and the probability distribution on the surface is proportional to $w_{\text {nucl }}(\alpha) \tilde{\mathcal{V}}(\alpha) \nu_{\text {civ }}(\alpha)$.

The functions $B(\alpha)$ and $\tilde{\mathcal{V}}(\alpha)$ depend on the choice of the time variable $t$ which is used to implement the cutoff. For example, if instead of the proper time we chose $\tilde{t}=\mathcal{V}_{*}(\alpha, t)$, 
then by construction the factor $\mathcal{V}_{*}$ would be the same for all universes. Here, we shall keep the proper time cutoff, which has a simple geometric and physical meaning. It favors the universes producing the largest number of civilizations per unit time by the clocks of the co-moving observers. The cutoff-dependence of the results is nontheless an important issue and requires further study [24].

The fractal dimension $d(\alpha)$ increases as the potential $V(\varphi)$ becomes flatter [21, 23], and thus the condition (4) selects maximally flat potentials with the highest value of $V_{\max }$. In some models, maximization of $B(\alpha)$ may drive the slope of $V(\varphi)$ to zero; then no reasonable cosmology is obtained. The approach presented here can be meaningful only if the maximum of $B(\alpha)$ corresponds to a non-trivial potential $V(\varphi)$. If we assume in addition that this maximum is degenerate and defines a surface rather than a single point, then the probability maximum on that surface is determined by the same considerations as in the case of finite inflation. In particular, the electroweak scale should not exceed the thermalization temperature, since otherwise no baryons would be formed. A more detailed discussion of $d \mathcal{P}(\alpha)$ in the case of eternal inflation will be given elsewhere.

Let us now summarize the "predictions" of the Principle of Mediocrity. The preferred models have very flat inflaton potentials, thermalization and baryogenesis at the electroweak scale, density fluctuations seeded by topological defects and a non-negligible $\Omega_{\Lambda}$ (as long as these features are consistent with one another and with the constraint (4) in the case of eternal inflation).

After this work was completed, I learned about the preprints by A.Albrecht [25] and by J.Garcia-Bellido and A.Linde [26] which have some overlap with the ideas presented 
here. I am grateful to Brandon Carter and Alan Guth for discussions and to Thibault Damour for his hospitality at I.H.E.S. where this work was completed. This research was supported in part by the National Science Foundation. 


\section{References}

[1.] B. Carter, in I.A.U. Symposium 63, ed. by M.S. Longair (Reidel, Dordrecht, 1974); Phil. Trans. R. Soc. Lond. A310, 347 (1983); B.J. Carr and M.J. Rees, Nature 278, 605 (1979); J.D. Barrow and F.J. Tipler, "The Anthropic Cosmological Principle" (Clarendon, Oxford, 1986). It should be noted that the Anthropic Principle, as originally formulated by Carter, is more than a trivial consistency condition. It is the requirement that anthropic constraints should be taken into account when evaluating the plausibility of various hypotheses about the physical world.

[2.] S. Weinberg, Phys. Rev. Lett. 59, 2607 (1987)

[3.] S. Coleman, Nucl. Phys. B307, 867 (1988).

[4.] E. Baum, Phys. Lett. B133, 185 (1984); S.W. Hawking, Phys. Lett. B134, 403 (1984).

[5.] S. Coleman, Nucl. Phys. B310, 643 (1988). In this paper Coleman obtained a probability distribution for $\rho_{v}$ with an extremely sharp peak at $\rho_{v}=0$. However, his derivation was based on Euclidean quantum gravity, which has serious problems. For a discussion of the problems, see W. Fischler et. al., Nucl. Phys. B327, 157 (1989).

[6.] L. Smolin, Class. Quant. Grav. 9, 173 (1992); Penn. State Preprint, unpublished.

[7.] T. Rothman and G.F.R. Ellis, Quart. J. Roy. Astr. Soc. 34, 201 (1993). 
[8.] A. Vilenkin, Phys. Lett. 117B, 25 (1982), Phys. Rev. D30, 509 (1984);

J. Hartle and S.W. Hawking, Phys. Rev. D28, 2960 (1983); A.D. Linde, Lett. Nuovo Cim. 39, 401 (1984).

[9.] A. Vilenkin, Phys. Rev. D27, 2848 (1983).

[10.] A.D. Linde, Phys. Lett. B175, 395 (1986).

[11.] We may wish to assign a weight to each civilization, depending on its lifetime and/or on the number of individuals. This would not change the conclusions of the present paper.

[12.] See, e.g., A.A. Starobinsky, Phys. Lett. 91B, 99(1980).

[13.] Including the vacuum contributions of matter fields to the expectation value of $T_{\mu \nu}$ and assuming slow rollover conditions, $\dot{\varphi}^{2} \ll V(\varphi)$ and $\dot{H} \ll H^{2}$, the evolution equation for $H$ takes the form

$$
H^{2}=\frac{8 \pi}{3 m_{p}^{2}} V(\varphi)+\frac{H^{4}}{H_{0}^{2}}-\frac{6}{M^{2}} H^{2} \dot{H}
$$

Here, $H_{0} \sim m_{p} / \sqrt{N}, N$ is the number of matter fields with masses $m \ll H$, and $M$ can be adjusted to any value by a finite renormalization of the quadratic in curvature term in the gravitational Lagrangian. Physically reasonable models are obtained for $H_{0}^{2}>0, M^{2}>0$ (for details see Ref. [12]). Classical inflationary solutions must have $\dot{H}<0$. This gives a quadratic inequality for $H^{2}$, which can be satisfied only if $V(\varphi) \leq 3 H_{0}^{2} m_{p}^{2} / 32 \pi$. The expansion rate cannot exceed $H_{0}$. A detailed discussion of this issue will be given elsewhere. 
[14.] Linde et. al. [23] have argued that the inflationary expansion rate is bounded by $H_{\max } \sim m_{p}$, because at this rate quantum fluctuations in the energymomentum tensor $T_{\varphi}^{\mu \nu}$ of the inflaton field $\varphi$ become comparable to $T_{\varphi}^{\mu \nu}$ itself, and the vacuum form of $T_{\varphi}^{\mu \nu} \propto g^{\mu \nu}$ is destroyed. I disagree with this argument. At $H \sim m_{p}$, quantum fluctuations in $T^{\mu \nu}$ for all fields with $m \ll m_{p}$ have comparable magnitude. The average total energy-momentum tensor is $\left\langle T^{\mu \nu}\right\rangle \propto g_{\mu \nu}$, and its relative fluctuation is $\sim N^{-1 / 2}$, where $N$ is the number of fields with $m \ll m_{p}$. Since $N \gtrsim 100$, the vacuum form of $T^{\mu \nu}$ holds with a good accuracy.

[15.] A. Vilenkin, Phys. Rev. D37, 888 (1988).

[16.] $\quad w_{\text {nucl }}$ depends also on the initial value of $\varphi$ and is maximized at $V(\varphi)=V_{\max }$.

[17.] For a review of topological defects, see A. Vilenkin and E.P.S. Shellard, "Cosmic Strings and Other Topological Defects" (Cambridge University Press, Cambridge, 1994).

[18.] G. Lazarides, C. Panagiotakopoulos and Q. Shafi, Phys. Rev. Lett. 56, 432 (1987); Phys. Lett. 183B, 289 (1987).

[19.] S.M. Carroll, W.H. Press and E.L. Turner, Ann. Rev. Astron. Astrophys. 30, 499 (1992).

[20.] A.A. Starobinsky, in "Current Topics in Field Theory, Quantum Gravity and Strings", ed. by H.J. de Vega and N. Sanchez (Springer, Heidelberg, 1986).

[21.] M. Aryal and A. Vilenkin, Phys. Lett. B199, 351 (1987)

[22.] Y. Nambu and M. Sasaki, Phys. Lett B219, 240 (1989) 
[23.] A.D. Linde and A. Mezhlumian, Phys. Lett. B307, 25 (1993); A.D. Linde, D.A. Linde and A. Mezhlumian, Phys. Rev. D49, 1783 (1994).

[24.] The dependence of $\rho(\varphi, t)$ on the time parametrization has been emphasized by Linde et.al. [23] and by Garcia-Bellido et.al. [27] who studied the probability distribution for the inflaton and other fields in a single eternally inflating universe.

[25.] A.Albrecht, Imperial College Report TP/93-94/56 (unpublished).

[26.] J. Garcia-Bellido and A.D. Linde, Stanford University Report SU-ITP-94-24 (unpublished)

[27.] J. Garcia-Bellido, A.D. Linde and D.A. Linde, Phys. Rev. D50, 730 (1994) 